\title{
PROPOSED BIOLOGICAL EXPLORATION OF MARS BETWEEN 1969 AND 1973
}

\begin{abstract}
A
STUDY ontitled "Biology and the Exploration of Mars" was convened by the Space Science Board of tho U.S. National Acaderny of Seiences-National Research Couneil during the summer of 1964, at the request of the National Aeronauties and Space Administration. It was conducted under the chairmanship of Prof. Colin S. Pittendrigh of Princeton University with Prof. Joshua Lederberg of Stanford University as cochairman. A list of participants and contributors has been published by the National Academy of Scionces. Meetings were held on the campus of Stanford University and at the Rockefoller Institute. A complete report is in preparation. Mcunwhile, Prof. Pittendrigh presonted tho results of this Study at the annual meeting of the Academy on April 26 : the substance of this roport follows here.
\end{abstract}

\section{Introduction}

Until recent years the origin of life and its possiblo ocsurrence olsewhere in the universe have boen matters for spectulation only. The rapid growth of molecular biology since 1940 has, to be sure, changed the discussion of life's origins into far more precise and explicit torms than were possible earlier; and the subjoct entered a new, experimental, phase in the $1950 \mathrm{~s}$ with successful abiogonic synthesis of important biochemical substances in conditions simulating the presumptive environment of the primitive Earth. But the real transformation which the subject has undergone stems from the spectacular growth of space technology in the past decade. The possibility of life's origin and occurrence on planets other than ours is no longer limited to idle speculation: it has entered the realm of the testable, of science in the strict sense. Given the rockets now available and especially those available by 1969 , it has become fully realistic to consider plans for the biological exploration of Mars.

The working group of this Study comprised 36 pooplo representing a broad spectrum of sciontific interests: ovolutionary biology, genetics, microbiology, biochemistry and molecular biology, animal physiology, soil chemistry, organic chemistry, planetary astronomy, geochemistry, and thoorotical physics. The membership included some with considerable prior involvement in probloms of space exploration and others with none. Advice was also sought outside the group of immediate participants on the potentialities of selocted analytical methods for the experimontal investigation of extra-terrestrial life and its environment. More than 30 individuals contributed in this fashion written assessments of techniques in which they wore particularly well versed.

Our task was to examine the scientific foundations and merits of the proposal to undertake a biological exploration of Mars. What were the potential scientific yields? How valuable, if attained, would they be? What, in fact, is the possibility of life occurring on Mars? And of our detecting it with the available and foreseoable technology? What could be achieved by further astronomical work from Earth; by Martian fly-by missions; by Martian orbiters; and Martian landers? What payloads would wo recommend for planetary missions? What timing and overall strategy would we recommend for Martian exploration wero we to consider it worth while at all ?

In brief the overall purpose was to rccommond to the Government through the Academy's Space Science Bourd, whether or not a biological exploration of Mars should be included in the nation's space programme over the next few decades; and, further, to outline what that programme, if any, should be.

Wo emphasize that our conclusions were reached on strictly scientific grounds; that we recognize a much wider array of considerations bear on any ultimato decision to undertake Martian exploration. As a body we were not charged with, nor did we attempt, the broad over-view which entnils these other considerations. We predicated our discussion on the continued vigour of a national space programme. We did not, for example, address oursolves to the question of whether the very large cost of developing the Saturn boostors could be justified on soientifie grounds. Nor should we havo; the development of the Saturn boosters is already firmly committed for other reasons. The questions we faced were whether the application of such boosters to the biological exploration of the solar systom-of Mars in particular-can answer well-framed and important scientific questions; and what priority these questions merit within the spaco programme.

The essence of our conclusions is that the exploration of Mars-notivated by biological questions-does indeod merit the highest scientific priority in the nation's space programme over the next decades. We concluded, further, that the favourable opportunities for exploration between 1969 and 1973 can and should be exploited as vigorously as possible. Considering the diversity of discipline and persuasion represonted by so large a group as ours, the unanimity achieved on these basic conclusions itself merits emphasis.

We achieved, understandably, less unanimity on precisely what course the exploration should take. 'There was a small minority among us which insisted that the first opportunity be taken to land a mission on Mars equipped with even a minimum of analytical devices. The majority view leaned towards a more gradualistic approach. Its foundation was two-fold: (1) the tenet that specifically biological questions should be asked in an ordered sequence of exploration the purpose of which is to understand the overall evolution of the planot's crust and atmosphere; (2) the large amount of work that remains to be done in designing and equipping a land device with the diversity of sensors necessary for study of a life of uncertain characteristies. The programme emerging from this approach begins with emphasis on orbiter missions designed to cnlarge our knowledge of tho astronomical, geophysical and geochomical features of Mars and its seasonal changes. Such new knowledge of the Martian environment will greatly enhance chances for the success of subsequent landing missions. Moreover, it will also pormit, prior to landing, a substantial re-evaluation of all those features on which our present judgment of the plausibility of Martian lifo depends.

This Study was prompted by a specific request from the National Aeronautics and Space Administration to the National Academy of Sciences. But in another way it has only boen the natural culmination of a discussion of many years in which many of the members of the working group have participated. To the extent it has succeeded in its assigned mission, it thus depends on the work of scveral carlicr committees and smaller symposia; and the conclusions and recommendations it presents are the product of prolonged deliberation and scrutiny, None of the working group failed to sense the burden of rosponsibility which such a costly programmo as Martian exploration entails; and none of them fuiled to sense the magnificent challenge and opportunity that are now before scientific man. 


\section{(1) Origin and Nature of Life}

The modern, naturalistic view of life's origin and evolution dates from the foundations of modern biology a century ago. Implicit in the evolutionary treatment of life is the proposition that the first appearance of organisms was only a chapter in the natural history of the planet as a whole. Oparin later made this notion explicit in his view that the origin of life was a fully natural, perhaps inevitable, step in the ontogeny of the Earth. Systems capable of self-replication and controlled transfer of energy-living organisms-had their origin in the sequence of chemical changes which were part of this planet's early history.

The tractability of this great inductive step to further discussion has been enhanced by the progress of terrestrial cellular biology and biochemistry over the past few decades. What has emerged from that progress is a unified picture of life at the sub-cellular and chemical levels, underlying the unity at higher levels which so largely influenced Darwin. Not only is there a common pattern to the structure of cellular organelles - membranes, mitochondria, nuclear apparatus, etc.--but also a still more surprising unity is found in its molecular constituents. Everywhere on Earth the essential catalytic functions are discharged by proteins, energy-transfer effected by ATP, and the synthesis of proteins to-day controlled by an elaborate nucleic acid system. The same enzymatic co-factors are found in organism after organism; particular metabolic pathways recur from cell to cell; and everywhere the fundamental functions of information storage and replication are assigned to the nucleic acids.

To a significant extent the discussion of life's origin must concern the origin of those molecular types which are crucial in cellular organization: the origin of nucleic acids, of proteins, of carbohydrates, and so on.

In the $1950 \mathrm{~s}$ a series of experiments was initiated in which the synthesis of biologically important compounds was accomplished by application of energy to presumptive primitive environments. The list includes: amino-acids and their polymers; carbohydrates and fatty acids; purines and pyrimidines; nucleotides, including adenosine triphosphate, and oligonucleotides-every major category of molecular sub-unit of which the cell is built.

The credibility of the naturalistic, evolutionary view of life's origin as an exploitation of previous chemical evolution on a sterile Earth is greatly heightened by these results: the great chemical complexity of its molecular constituents does not, in the last analysis, require the intervention of the cell itself.

The general tenet that life involves no qualitative novelty-no élan vital - goes hand in hand with the more explicit proposition that it is the molecular organization, as such, of living things which alone distinguishes them from the non-living. The central issue in discussing origins now concerns not so much the prior evolution of complexity in molecular constituents as the attainment of their organization into a system which is alive. It is here we lack any sure guides - except one-on the contingency involved; on how improbable it all was. That one lead comes from the great and well-known advances of molecular genetics in the past ten years.

The essence of organization in one sense is its improbability, its dependence on specification or information: and the most characteristic feature of living organizationsorganisms-is their capacity to store and replicate the evolving information on which their existence depends. The high point of our biochemical advance has been identification of the molecular basis of these defining characteristics. It is astonishing how much we have recently learned about the manner in which the information underlying life's organization is encoded in molecular structure; that we understand how that molecular structure is replicated; and further, that simple polynucleotides have been synthesized in cell-free systems.

It is still not clear, of course, what precise sequence of events exploited the opportunities afforded by the purely chemical evolution of the Earth's surface and atmosphere. But at some point in the unknown sequence a community of molecules would have been fully recognizable to us as a living as against a non-living thing: it would have been bounded from its environment by a membrane, capable of controlled energy expenditure in fabricating more of itself and endowed with the capacity to store and replicate information.

We cannot fully know the precise course of the Earth's early chemical evolution, and the degree of contingency involved in the subsequent transition to a living organization of molecules; and for these reasons we cannot fully assess just how probable or improbable life's origin was at the outset of our own planet's evolution. Nor can we estimate to what extent the emerging picture of a single chemical basis to life on Earth reflects a physical necessity for living organization as against a mixture of physical sufficiency and historical accident. Can the catalysis, essential to biochemical organization, be effected only by proteins containing the 20 amino-acids we encounter in cells? Are the nucleic acids the only polymers, for physical reasons, which can carry molecular information on satisfactorily? Or are these and other empirical generalizations about life on Earth, such as optical activity, merely reflexions of the historical contingency which gave such molecules first access to living organization, thus pre-empting the field and precluding realization of other physically sufficient molecular foundations for life?

To the extent that we cannot answer these questions we lack a true theoretical biology as against an elaborate natural history of life on this planet. We cannot prejudge the likelihood of life's appearance on Earth; therefore we cannot confidently take the great inductive step when we are told by astronomers that there may be $10^{20}$ planetary systems elsewhere in the universe with histories comparable to our own. One thing is clear-if life is unique to our planet the probability of its origin must be almost unimaginably low. If, on the other hand, the probability is at all appreciable, life must be abundant in the $J 0^{20}$ planetary systems which fill the sky.

What is at stake in this uncertainty is nothing less than knowledge of our place in Nature. It is the major reason why the sudden opportunity to explore a neighbouring planet for life is so immensely important.

We emphasize that the act of discovery itself would have this great scientific, and for that matter philosophical, impact. But it is also important that discovery would, in another way, be only the beginning. The existence and accessibility of Martian life would mark the beginning of a true general biology, of which the terrestrial is a special case. We would have a unique opportunity to shed new light on the meaning of that astonishing molecular similarity in all terrestrial organisms. Is it there as a physically necessary basis for life? Or is itphysically sufficient but not necessary-a historical accident in the sense that in another instance of planetary evolution a different basic chemical complexity could equally well have emerged and pre-empted the local opportunity for life?

\section{(2) Possibility of Life on Mars}

No thoughtful person will disagree with our assertion on the scientific importance of life elsewhere in the Solar System. It is, however, another matter to conclude that the search for it should proceed at once. The exploration will be costly in money and other resources. To undertake it we need some assurance it is not folly from the outset.

Interest immediately focuses on Mars. The nearest and most Earth-like of the planets in the Solar System 
are Mars and Venus; but the surface of Venus has been tentatively excluded as a possible abode of life, because of the probably high surface temperatures. The Martian year is long ( 687 days) but the length of its day is curiously similar to that of Earth, a fact that to a considerable degree ameliorates an otherwise very severe environment.

Mars has retained an atmosphere, although it is thin: present estimates of pressure at the surface range from 10 to 80 millibars. The major constituents are unidentified, but are thought to be nitrogen and argon. Carbon dioxide has been identified spectroscopically and its proportion estimated to lie between 5 and 30 per cent by volume. Oxygen has been sought but not detected; the sensitivity of measurement implies a proportion not greater than 0.1 per cent by volume. Water vapour has also been identified spectroscopically as a minor atmospheric constituent to the amount of $2 \times 10^{-3} \mathrm{~g} \mathrm{~cm}^{-2}$. (For comparison, approximate terrestrial values of the quantities given above are: surface pressure 1,000 millibars; carbon dioxide 0.03 per cent; oxygen 20 per cent; water vapour $3 \mathrm{~g} \mathrm{~cm}^{-2}$.)

The intonsity of ultra-violet radiation at the Martian surface may be high by comparison with Earth, but this is not yet certain; some models of the composition of the atmosphere allow for effective shielding.

Surface temperatures overlap the range on Earth: at some latitudes and seasons they have a daily high of $+30^{\circ} \mathrm{C}$ with a diumal range of about $100^{\circ} \mathrm{C}$.

There are two white polar caps the composition of which has been the subject of some controversy. The evidence is now clear that they are ice, in the form of hoar frost. They undergo a seasonal waxing and waning which is probably accompanied by an atmospheric transfer of water vapour from one hemisphere to another.

Our knowledge of what lies between the polar caps is limited to the distinction between the so-called 'dark' and 'bright' areas and their seasonal changes. The latter, usually considered 'deserts', are an orange-ochre or buff colour. The former are much less vividly coloured. It is likely that early descriptions of the dark areas as green resulted from an optical illusion due to contrast with the orange 'bright' areas.

Biological interest nevertheless continues to centre on the 'dark' areas. In several respects they exhibit the kind of seasonal change one would expect were they due to the presence of organisms absent in the 'bright' (desert) areas. In spring the recession of the ice cap is accompanied by development of a dark collar at its border, and as the spring advances a wave of darkening proceeds through the dark areas toward the equator and, in fact, overshoots it $20^{\circ}$ into the opposite hemisphere.

Polarimetric investigations suggest that much of the Martian surface may be covered with small sub-millimetresized particles. The curve on which this inference is based shows a seasonal displacement in the dark areas, but not in the bright. Infra-red absorption features have been attributed to the dark areas, suggesting abundant $\mathrm{H}-\mathrm{C}$ bonds there, but more recent analysis throws great doubt on this interpretation, leaving us with no definite information, one way or the other, about the existence and distribution of organic matter.

Needless to say, none of these inferences about the Martian dark areas demands the presence of organisms for their explanation.

Indeed, the question is whether the Martian environment could support life at all; and further, whether its history would have permitted the indigenous origin of life. These are clearly different questions. Our answer to the first question is that we find no compelling evidence that Mars could not support life even of a kind chemically similar to our own. Were oxygen present to the small limiting extent current measurements allow, a fully aerobic respiration would be possible. But even its total absence would not of itself preclude life. One of our more rewarding exercises has been the challenge to construct a Martian ecology assuming the most adverse conditions indicated by present knowledge: it posed no insuperable problem. Some terrestrial organisms have already been shown to survive freeze-thaw cycles of $+30^{\circ}$ to $-70^{\circ} \mathrm{C}$. Others are known to cope with extremely low humidities and derive their water supply metabolically. There are many conceivable ways of coping with a strong flux of ultra-violet (and even of exploiting it as an energy source). The history of our own planet provides plenty of evidence that, once attained, living organization is capable of evolving adjustments to very extreme environments. And, finally, we are reminded that the evidence we have on Martian conditions is very coarse-grained, a sort of average that takes account of almost no local variations dependent on topography. Within the range of conditions represented by our present numerical estimates it is likely that there exist, perhaps abundantly - as on Earth-places where the extremes of temperature, aridity, and adverse irradiation are markedly ameliorated. Even the presence of water in the liquid phase is perhaps not unlikely, if only transiently, by season, in the sub-soil.

A measure of our judgment that niches in the contemporary Martian environment could support life of a sort comparable with that of Earth is provided by our overriding concern with the danger of inadvertently contaminating Mars with terrestrial organisms. We shall return to this problem later.

The other question--whether life in fact is there-depends on our judgment of how probable its origin on Mars has been. The a priori probability of origin we can not assess, even for Earth; it is the principal reason for considering exploration in the first place.

Given all the evidence available at present, we believe it entirely reasonable that Mars is inhabited with living organisms and that life independently originated there. However, it should be clearly recognized that our conclusion that the biological exploration of Mars will be a rewarding venture does not depend on the hypothesis of Martian life. The scientific questions which ought not to be prejudged are: $(a)$ Is terrestrial life unique? The discovery of Martian life, whether extant or extinct, would provide an unequivocal answer. (b) What is the geochemical (and geophysical) history of an Earth-like planet undisturbed by living organisms? If we discover that Mars is sterile we may find answers to this alternative and highly significant question.

\section{(3) Scientific Aims of Martian Exploration}

We approach the prospect of Martian exploration as evolutionary biologists. The origin of organisms was a chapter in the natural history of the Earth's surface. The hypothesis to be tested is a generalization from that single case: the origin of living organization is a probable event in the evolution of all planetary crusts which resemble ours. We thus conceive the over-all mission as a systematic investigation of the evolution of the Martian surface and atmosphere: has that evolution included, in some niches of the planet, chemical systems with the kind of organization we would recognize as 'living' ?

Our aims in summary form are: (1) The determination of the physical and chemical conditions of the Martian surface as a potential environment for life; (2) the determination whether or not life is or has been present on Mars; (3) the characteristics of that life, if present; (4) investigation of the pattern of chemical evolution without life.

This formulation emphasizes that as biologists we have as much interest as the planetary astronomers in a thorough investigation of the meteorology, geochemistry, geophysics, and topography of Mars. Whatever the outcome of a direct search for life, its full meaning will escape us unless the findings can be related to the prevailing environment. 


\section{(4) Avoiding the Contamination of Mars}

Before proceeding to the more programmatic aspects of the undertaking, we are concerned to single out the task of spacecraft sterilization from the many and diverse problerns that Martian exploration will entail. We believe that many of our non-biologist colleagues have still not fully grasped either the magnitude or the fundamental importance of this issue.

Contamination of the Martian surface with terrestrial microbes could irreversibly destroy a truly unique opportunity for mankind to pursue a study of extra-terrestrial life. Other future uses of Mars are not evident to us now; whatever they are, they may be clumsily destroyed by premature and uninformed mistakes in our programme. Wo are eager to press Martian exploration as expeditiously as the technology and other factors permit. However, our present sure knowledge of Mars is very slim, and so our recommendation to proceed is subject to one rigorous qualification: that no viable terrestrial micro-organism reach the Martian surface until we can make a confident assessment of the consequences.

In operational context this means that the probability of a single viable organism reaching the Martian surface be made small enough to meet scientifically acceptable standards. These standards, already established provisionally*, should be continually re-examined in the light of all new information. Moreover, every effort should be made to ensure the continued acceptance by other launching nations of the recommended confidence-levels for protection of Mars against contamination. The technical problems precipitated by this demand include the control of trajectories to an accuracy sufficient to prevent the accidental impact of unsterilized payloads, the development of sterilizable spacecraft components for vehicles intended for landing, the development of procedures which will prevent the introduction of microorganisms and the means for establishing the reliability of the entire programme. Since we have not yet succeeded in sterilizing a space-vehicle, the problem must be considered unsolved.

An energetic programme for the development of sterilization procedures of space vehicles and their components must be implemented immediately if we are to take advantage of the opportunities which will arise during 1969-73. We must guard not only against accidental neglect of necessary safeguards but also against placing ephemeral considerations of prestige above enduring scientific significance and utilitarian value in our exploration of space.

\section{(5) Avenues of Approach to the Exploration of Mars}

For convenience, we distinguish four categories of work that can contribute to attaining our goals: $(a)$ laboratory work needed to develop techniques for planetary investigations and the knowledge needed to interpret their findings; $(b)$ Earth-bound astronomical investigations of Mars; $(c)$ the use of spacecraft for the remote investigation of Mars; (d) a direct study of the Martian surface by landing missions.

(a) Laboratory Work. The consideration of the evolution of life on Mars raises many problems which can be investigated in Earth-based laboratories. Such investigations are, in fact, essential to provide the background against which the results of planetary missions must be interpreted. The work includes the chemical analysis of meteorites, especially with respect to their content of organic compounds, and the extension of investigations of the spontaneous formation of organic molecules and their aggregation into larger units. These investigations may reveal to us the mechanism by which not only the

* Report of Seventh Meeting of the Committee on Space Research, Florence, Italy, May 1964, Resolution 26. materials essential for living organisms were first formed, but also the origin of reactions and mechanisms that lead to the formation of organized structures and their selfperpetuation. Other possibly interesting lines of effort include alternatives to the carbon-water system of biochemistry and simulations of Martian and other planetary environments. While some of these simulated environments may allow terrestrial micro-organisms or enzyme systems to function, others may be more conducive to the activity of reaction systems based on alternative biochemistries.

It will become clear later that considerable work remains to be done in defining schemes for life detection and in developing the instrumentation to exploit them.

(b) Earth-bound Astronomical Studies of Mars. The observation of Mars from terrestrial observatories enjoys the advantages of economy, absence of weight and size limitations, and high data rate. It is, however, limited by the terrestrial atmosphere in attainable resolution and spectral range and further constrained by daylight and weather. Nevertheless, much valuable work could be conducted at a cost which is low compared to that of space programmes if the nation's large instruments were made available during prime seeing time for the observation of Mars. The use of 120 -in. and 200 -in. optical telescopes and of tho largest radio telescopes and interferometers could rapidly extend our knowledge of Mars. We support the recommendations of another committee of the National Academy of Sciences $\dagger$ on the need for additional ground-based astronomical facilities. For such facilities to play a significant part in the planning of 1969-73 Mars missions work on this programme must be begun early.

(c) Use of Spacecraft for Remote Observation of Mars. Some of the observational limitations imposed by the terrestrial environment can be overcome by balloon-borne observatories, but since they are severely restricted in size and observation time their usefulness is limited; it is also restricted by absorption in the Earth's atmosphere. The projected Earth-orbiting astronomical observatory $(O A O)$ overcomes some of these limitations and we believe the observation of Mars, particularly in the ultraviolet, should be ineluded in the plans for its use.

It is, however, from Martian fly-by missions and, in particular, from Martian orbiters that the remote obseivation of that planet is best undertaken. We hope to obtain our first close-up information on the Martian surface from the video scan to be carried out by Mariner $I V$ and to gain additional knowledge of atmospheric density by observation of the telometry signals during occultation of the spacecraft.

Fly-by missions are, however, severely limited in the time available for observation; they provide at best a floeting glimpse of the planet.

Martian orbiters will be technically possible for the opportunities of 1969 and thereafter. They offer an unparalleled opportunity to scrutinize the planet at comparatively short range. Potential orbiter payloads have been examined by another group and compositions of such payloads have been suggested for a range of instrument weights up to $200 \mathrm{lb}$. (which is within the capability of the Saturn IB-Centaur). For example, a modest payload which any of several vehicles could place in orbit could include instruments for: (1) infra-red and television mapping; (2) microwave radiometry and bistatic radar; (3) infra-red spectrometry; (4) optical polarimetry. These sensors would yield information on temperatures, surface and atmospheric composition, topography, certain characteristics of surface structure, etc., and, most important of all, permit a sustained scrutiny through a full cycle of seasonal change and over a major fraction of the Martian surface.

$\dagger$ Ground-based Astronomy, A Ten-Year Programme, National Academy of Sciences Publication No. 1234 (1964). 
(d) Martian Landing Missions: ABLs Small and Large. While it is conceivable that the findings of a Martian orbiter could establish the presence of life on the planet, we are in any event convinced that landing missions are essential for adequate Martian exploration. The definition of lander payloads is a complex and demanding task which we have only begun to explore.

Their design is to some extent dependent on our knowledge of the structure of the Martian atmosphere. The size of the payload that can be deposited depends, for example, on whether the use of a parachute is feasible or whether the density of the atmosphere is so low as to require the use of retrorockets--this is especially critical for small payloads. In this connexion, we note the possibility that the density profile of the Martian atmosphere will be determined by astronomical means, or by Mariner IV, with sufficient precision for the purpose of designing a landing system. A more direct method for studying the Martian atmosphere involves the use of non-survivable atmospheric entry probes that could transmit information on atmospheric density structure and composition. Such probes could be launched from either fly-bys or orbiters. Since their design is not dependent on atmospheric density, these are useful devices for obtaining advance information, if needed, for the survivable landing of an instrument package. The view has also been presented that a small surviving capsule would have even more value, in that it might determine not only the density profile of the atmosphere but also its composition at the surface, wind velocity and other data that would enhance the probability of success of a large lander.

However, if we had a complete knowledge of these pre-requisites for a successful survivable landing device, our principal design difficulty would remain: it concerns the problem of life-detection. What minimal set of assays will permit us to detect Martian life if it does exist ? A debate on this question for the past several years has yielded a variety of competing approaches. Each of these is directed to some manifestation of life according to the cues of terrestrial biology. Needless to say, visual reconnaissance, from microscope to telescope, is one of the most attractive of these, for it offers the expectation that many recognizable hints of life would immediately attract our attention. However, we can easily imagine circumstances in which this type of observation would be inconclusive. Many other suggested procedures seek to identify, at the outset, the more fundamental biochemical structures and processes that we would, in any case, explore in depth. No one of these analyses, however, whether photosynthesis or respiration, DNA or proteins, growth, enzymes or metabolism, or, in a figurative sense, fleas or elephants, can be sure of finding its target and reliably reporting on it under all circumstances, nor would any single approach satisfy all the particular interests that motivate different investigators in their search.

We cannot recount here all our deliberations on the life detection problem. We have sought the most generalized criteria; among these is net optical activity, which is almost surely the result of steric restrictions imposed by an historical accident in the origin of life. Another is the presence in assays of exponential features which can only be ascribed to growth and reproduction. And we have reconciled ourselves to the fact that early missions should assume an Earth-like carbon-water type of biochemistry as the most likely basis to any Martian life. On that assumption enzymes that should be widespread can be sought and growth may be demonstrable by the use of generalized media [see \& lso p. 1019 of this issue].

The fact remains, and dominates any attempt to define landers for detecting life, that no single criterion is fully satisfactory, especially in the interpretation of some negative results. To achieve the previously stated aims of Martian exploration we must employ as mixed a strategy as possible.
Diseussion throughout our study has returned repeatedly to the conclusions that we would not be convinced by negative answers from single 'life-detectors', that given the hazards of any chemical or metabolic assay we should ensure some direct visual inspection by television, and that the lander programme must ultimately involve an automated biological laboratory (ABL). The ABL concept is not fully defined: it involves provision for the multiplicity and diversity of chemical analytical techniques and biological assays that our aims call for; it involves, too, the idea of an on-board computer by means of which a variety of programmed assay sequences can be initiated contingently on the results of prior steps; it also involves the idea of a sustained discourse between the computer and investigators on Earth. It is, in short, an ambitious concept. But our preliminary scrutiny of the $A B L$ idea suggests that, though ambitious, it is, in principle, realizable with the current technology.

In the long run we believe that manned expeditions and the return of Martian samples to the Earth will be part of the exploration of the planet. Neither of these is imminent, but some of our readers will be as surprised as we were to discover that manned Martian missions will probably be feasible in the 1980 s. Certainly neither the return of samples nor the sending of men to Mars will be scientifically justifiable until unmanned landings have prepared the way.

\section{(6) Timing and Overall Strategy of Explorations}

All of us would in principle prefer a gradualistic approach to the ultimate goals of landing a large $\mathrm{ABL}$ on Mars and, eventually, of returning samples for study here. It is clear on all grounds-of economy and scientific prudencethat we should exhaust the possibilities of further progress using Earth-based observations and non-landing missions to Mars.

For example, a strong majority of the working group believes a successful orbiter programme should precede a landing. The orbiter promises an immense extension of our knowledge of the atmosphere (its density and chemical composition) and surface of Mars. Its capability for sustaining seasonal observation and extensive topographic mapping will permit a thorough re-evaluation of the several Martian features that have been considered suggestive of life. And it will permit a far better informed selectien of landing site for the ultimate ABL missions. It has the further merit of effecting this substantial step forward with minimum risk of contaminating the surface.

Constraints to proceeding in a completely unhurried, stepwise fashion arise from several sources, however. They are a combination of celestial mechanics and the operational realities of space research. Any space experiment takes years of preparation and budgetary commitment; the preliminaries to actual flight involve years of experimental design, spacecraft development, and the co-ordination of effort among large numbers of people in a wide range of disciplines. The scientific investigator no longer has the total freedom he usually enjoys to make tentative starts, to explore hunches without full commitment, to stop and follow another course. He is further plagued by the prospect of investing years of work only to encounter a mission failure or cancellation in which it is all lost--at least until a new opportunity arises perhaps years hence. He may chafe in these circumstances, but he must accept them if he wishes to proceed at all. The kind of Martian lander that we visualize will be a most complex and difficult spacecraft to build and will require the combined efforts of many different scientific specialists. It will be, for these reasons, most costly and time-consuming to develop. A Martian orbiter is also a much larger undertaking than any scientific spacecraft so far flown. The point is that we are confronted with the necessity of near-commitment many years ahead of flight time; and the opportunities 
for flights to Mars are by no means always at hand. The orbits of Earth and Mars are such that these opportunities are now limited to brief windows which recur about every second year but undergo a further approximately 17-year cycle of favourableness. Our attempt to develop a systematic and gradualistic programme is thus constrained to some extent by the fact that while favourable opportunities occur in the $1969-73$ period they will not return before 1984-85. (For these reasons an alternative strategy has been discussed: it would allow the early use of landing probes, always providing that reliable decontamination systems will have been developed and authenticated. A minority opinion holds that small landers may provide environmental information useful in the design of other spacecraft and may succeed more readily than orbiters. According to this view the way should be left open to their use-even though the results obtained may well be less comprehensive.)

We have concluded that the 1969-73 opportunities can be and should be exploited for a substantial programme of planetary missions. By that time the Saturn booster system will be available, and a four to five year lead time is evidently adequate for the development of initial spacecraft.

The more detailed planning of planetary missions for 1969-73 is for the most part outside the scope of this Study's competence and commission: the decisions concerned involve engineering and many other elements, with which we did not cope.

\section{(7) Conclusions and Recommendations}

(1) Biological Exploration of Mars Recommended. The biological exploration of Mars is a scientific undertaking of the greatest validity and significance. Its realization will be a milestone in the history of human achievement. Its importance and the consequences for biology justify the highest priority among all objectives in space science --indeed in the space programme as a whole.

(2) Scientific Aims of the Exploration. We approach the prospect of Martian exploration not only as biologists but also as scientists interested in evolutionary processes over the broadest range. Living systems have emerged as a chapter in the natural history of the Earth's surface. $W_{\theta}$ wish to test the hypothesis that the origin of life is a probable event in the evolution of all planetary environments the histories of which resemble ours.

We thus conceive the over-all mission as a systematic study of the evolution of the Martian surface and atmosphere: has that evolution included, in some niches of the planet, chemical systems with the degree of complexity, organization and capacity for evolution we would recognize as 'living' ? Our specific aims are: $(a)$ determination of the physical and chemical conditions of the Martian surface as a potential environment for life; (b) determination whether or not life is or has been present on Mars; (c) characterization of that life, if present; (d) investigation of the pattern of chemical evolution, in the absence of life.

(3) An Immediate Start to Exploit the 1969-73 Opportunities. A major effort should be initiated immediately to exploit the particularly favourable opportunities of 1969 to 1973.

We are here concurring with the U.S. Space Science Board's views that planetary exploration should be the major aim of the nation's space science efforts in the $1970 \mathrm{~s}$ and 1980s; and, further, that the biological exploration of Mars be the primary focus of the programme.

(4) Avoiding the Contamination of Mars: a Major Mission Constraint. Before proceeding to other aspects of the undertaking, wo are concerned to single out, from the many and diverse problems that Martian exploration will entail, the task of prevention of contamination.

Contamination of the Martian surface with terrestrial microbes could irrevoeably destroy a truly unique oppor- tunity for mankind to pursue a study of extra-terrestrial life. Thus, while we are eager to press Martian exploration as expeditiously as the technology and other factors permit, we insist that our recommendation to proceed is subject to one rigorous qualification: that no viable terrestrial micro-organisms reach the Martian surface unt il we can make a confident assessment of the consequences. (See Section 5 above.)

(5) Programme Recommendations. 5.1. Every oppoitunity for remote observation of Mars by Earth-bound or balloon and satellite-borne instruments should bo exploited. A vigorous programme here can yield a very substantial increase in our knowledge of Mars before the major programme of planetary missions begins in 1969.

5.2. It has become evident that an adequate programme for Martian exploration cannot be achieved without using scientific payloads substantially larger than those at present used in our unmanned space research programme. Although predominantly engineering considerations may incline to early use of smaller payloads, we see very substantial advantages in the use, from the outset, of the new generation of large boosters which are expected to become operational toward the end of the present decade. These advantages include: the possibility of avoiding spacecraft obsolescence due to a change in booster; the potential for growth in the versatility of scientific payloads and the relief of pressure on the engineer to design spacecraft to the limit of booster capacity.

5.3. We deliberately omit an explicit recommendation in favour of any fly-by missions additional to those already executed or planned for the 1964 (and possibly 1966) opportunities. They yield at best a fleeting glimpse of the planet, and unless they are already so large that they could as well have been orbiters, the array of sensors they carry is small. Given the booster power adequate to deliver it, an orbiter is overwhelmingly preferable. It. may well be, however, that strictly engineering con. siderations will demand some preliminary flights in 1969 and, if these are undertaken, their exploitation as fly-bys could yield worthwhile information.

5.4. Every effort should be made to achieve a large orbiting mission by 1971 at the latest. This mission should precede the first landing device. (A dissenting minority view supports the simultaneous use of small landing probes.) By 'large' we mean a scientific payload which would include instrumentation for: $(a)$ infra-red and television mapping; (b) microwave radiometry and bistatic radar; (c) infra-red spectrometry; $(d)$ optical polarimetry. The success of this mission will depend on the availability of a large booster and a substantial improvement in the communications facilities at present available.

5.5. The first landing mission should be scheduled no later than 1973 and by 1971 if possible.

We have not yet outlined what the contents of a large landing device should be in terms as specific as those used to deseribe the orbiter. The central point on which all agree is that the mission ultimately demands a large lander, which we have come to call an ABL (automated biological laboratory). What is not clear at present is how fast such a large landing device can be designed. and developed from biological and engineering points of view. It is, however, clear that the development, both as to conceptual design and engineering, will go through several generations. It is hoped that the first generation of an $A B L$ could be used for the 1971 opportunity.

The landing device we are recommending for 1971 is something short of what is ultimately possible and nocessary, but could have a sufficiently diverse array of instrumentation to answer some of the scientific questions we have posed.

5.6. The task of designing an ABL should be initiated immediately as a continuing project. The contents of 
landers in 1971 and 1973 will be products of this continuing undertaking.

5.7. The problems associated with the biological exploration of Mars are diverse and the task of implementation raises challenges in many respects wholly novel. Orbiter and landing missions alike will involve many different experimenters. The evolution of an optimum scientific payload will require a continuing dialogue among all potential investigators and the engineers responsible for implementing their scientific goals. The undertaking we are recommending cannot proceed without some provision for organizing and sustaining that dialogue on a continuing basis. As the programme develops, other devices may become more appropriate, but at the outset we believe a standing committee of the Space Science Board will be a useful provision. It should be charged with: (1) a continuing surveillance of progress from a scientific point of view; and (2) the responsibility of giving advice to the National Aeronautics and Space Administration.

\title{
ELECTROCHEMICAL MECHANISM OF ATMOSPHERIC RUSTING
}

\author{
By DR. U. R. EVANS, F.R.S. \\ Emeritus Reader in the Science of Metallic Corrosion, University of Cambridge
}

$\mathrm{T}$ HE electrochemical character of corrosion under wet conditions has long been established. On iron ${ }^{1,2}$, aluminium ${ }^{3}$ and zinc ${ }^{4}$ the current flowing between distinct anodic and cathodic areas has been found equivalent to the corrosion rate in the sense of Faraday's law. The case of atmospheric corrosion has received less attention, although Rosenfel'd ${ }^{5}$ has reported electrochemical reactions on metal covered with a thin film of liquid. The experiments described here were made on specimens introduced dry into moist (unsaturated) air containing sulphur dioxidethe type of atmosphere responsible for the familiar dark rust on steel; moist unpolluted air produces little damage.

The experiments refer to mild steel sheet and also relatively pure iron electro-deposited on steel, nickel or copper substrates; also to unplated nickel, where Vernon's observations $^{6}$ were confirmed. In the main series the relative humidity of the air was 95 per cent and the sulphur dioxide content was about $0 \cdot 2$ per cent-exaggerated conditions chosen for quick results. Similar changes were observed at lower contents of water and sulphur dioxide. In some experiments the $\mathrm{SO}_{2}$ content was reduced to (nominal) zero after a few days; rusting continued. A different sequence was observed at high $\mathrm{SO}_{2}$ contents, leading to the sulphide film already reported by Preston and Tholen?

On iron or steel the atmosphere mentioned produces change within $5 \mathrm{~min}$; a faint mist appears on the surface, which can be wiped off with filter paper; ferricyanide testing shows ferrous iron to be present. Later, dark particles appear on the metal surface; these also are partly removable on filter paper. Besides the dark particles, others, invisible against the paper, are shown up as blue points with ferricyanide; apparently they are micro-crystals of ferrous sulphate. The dark particles contain much water-soluble $\mathrm{Fe}^{2+}$ if the exposure has been short, but after several hours the amount of water-soluble iron declines. The acid-soluble material is $\mathrm{Fe}^{2+}$ and $\mathrm{Fe}^{3+}$ in the early stages, but mainly $\mathrm{Fe}^{3+}$ later, when a trace of water-soluble $\mathrm{Fe}^{3+}$ can be detected. The dark spots rapidly spread over the surface; after a day, steel is completely covered. The deposit is grey at first, but becomes red-brown; the colour change occurs more quickly when the $\mathrm{SO}_{2}$ content is low, although the amount of deposit is then smaller. Some of the rust is removable by gentle wiping, but the major part resists scrubbing with a stiff bristle brush.

It was found that, when once ferrous sulphate has appeared, rust-formation can continue even though the specimen is moved to moist air containing no sulphur dioxide; this appears to support the views put forward by Vernon ${ }^{8}$ and by Schikorr ${ }^{9}$ that rust is formed from the ferrous sulphate; evidently the ferrous sulphate is regenerated, since one atom of sulphur can produce many molecules of rust. They believe that rust is formed by oxidation and hydrolysis of ferrous sulphate and that the acid set free by hydrolysis then attacks more iron.

The new results confirm Vernon's view ${ }^{5,6}$ that at the outset iron behaves like nickel. Adsorbed sulphur dioxide is catalytically oxidized to trioxide which collects water from the unsaturated atmosphere; the sulphuric acid attacks the metal giving ferrous sulphate. (The attack of sulphuric acid on iron would to-day be regarded as electrochemical, the anodic reaction being the formation of $\mathrm{Fe}^{2+}$ and the cathodic reaction the reduction of oxygen. At high $\mathrm{SO}_{2}$ concentrations, the cathodic reaction may be its reduction to $\mathrm{S}_{2} \mathrm{O}_{4}{ }^{2-}$, as suggested by Rosenfel' ${ }^{5}$; sometimes, it appears, cathodic reduction proceeds as far as $\mathrm{S}^{2-}$, accounting for the sulphide films observed by Preston and Tholen ${ }^{7}$. Mayne ${ }^{5}$ considers that the cathodic reduction of $\mathrm{SO}_{2}$ only occurs at high concentrations. The new results confirm him, and are consistent with recent Russian opinion as summarized in the papers on "Corrosion of Metals and Alloys", collected by N. D. Tomashov (Moscow, 1963). It is stated that "with high sulphur dioxide concentrations $(0.5$ per cent and higher)" there is "stimulation of the cathodic process due to the oxidizing properties of the sulphur dioxide" (p. 414 of the English translation by A. D. Mercer, edit. by C. J. L. Booker).) Steel will be attacked much more quickly than nickel, and the ferrous sulphate formed will take up most of the moisture on the surface as water of crystallization, thus explaining a fact-observed 42 years ago ${ }^{10}$ - that steel remains merely damp in a sulphurous atmosphere which renders nickel running wet.

How is the rust produced from ferrous sulphate? The rust formed in the early stages, and the loose outer portions of that formed on longer exposure, probably arise from oxidative hydrolysis ${ }^{8,9}$; the detection of watersoluble $\mathrm{Fe}^{3+}$ confirms this. But the clinging rust can scarcely arise from hydrolysis; rust would not adhere strongly if the basis was constantly being eaten away. If, however, anodic attack is proceeding in crevices ( $A$ in Fig. 1), balanced by cathodic reduction of oxygen to $\mathrm{OH}^{-}$on the face $(C)$, ferrous hydroxide will be deposited on the cathodic area and will quickly be oxidized to the ferroso-ferric and ferric condition. Such a mechanism would explain the strong adhesion of the rust (comparable with the adhesion of electro-plated metal), and would provide for complete regeneration, since as many ferrous ions will be formed at the anodic points as are lost on the cathodic area.

An early research ${ }^{10}$ demonstrated electrochemical phenomena at bimetallic junctions on unwetted specimens. But in everyday rusting no bimetallic couple is present and, although on steel the couple iron/cementite might operate, this is not available on pure electro-deposited iron, which develops rust like steel. Probably the current is generated by differential aeration, iron being attacked 